\title{
Croissance et caractéristiques de carcasse de poulets sélectionnés en lignées divergentes soit sur la vitesse de croissance soit sur l'état d'engraissement
}

\author{
F.H. RICARD et B. LECLERCQ \\ avec la collaboration technique de G. MARCHE \\ I.N.R.A., Station de Recherches avicoles, Centre de Recherches de Tours \\ Nouzilly, F 37380 Monnaie
}

\begin{abstract}
Résumé
Des poulets de 4 lignées expérimentales, sélectionnées soit pour une vitesse de croissance forte ou faible (X-33 et X-44) soit pour un dépôt gras abdominal fort ou faible (《grasse» et "maigre 》), ont été élevés ensemble jusqu'à l'âge de 9 semaines. Les animaux ont été pesés aux âges de 3, 6 et 9 semaines et l'angle de poitrine a été mesuré sur le vivant à 9 semaines. Après abattage de 32 coquelets par lignée, on a procédé à la mesure de l'angle de poitrine sur la carcasse effilée et du dépôt gras abdominal.

Le choix des lignées étudiées entraîne des différences entre échantillons pour tous les caractères mesurés. Entre 3 et 9 semaines, la lignée maigre présente un gain de poids légèrement, mais significativement, supérieur à celui de la lignée grasse. Les corrélations avec le poids vif sont en moyenne de 0,994 pour le poids effilé, de 0,59 pour le poids du dépôt gras abdominal, de 0,40 pour le pourcentage de gras abdominal, de 0,29 pour le rapport poids effilé/poids vif et seulement de 0,26 pour l'angle de poitrine.

Les variations de poids de la carcasse effilée suivent strictement celles du poids vif et les 4 droites de régression du poids effilé sur le poids vif peuvent être confondues. Les variations de l'état d'engraissement font apparaître un comportement différent selon les lignées. Les corrélations avec l'angle de poitrine sont pratiquement nulles pour les lignées grasse et maigre et de l'ordre de 0,45 pour les lignées X-33 et X-44. Les coefficients d'allométrie poids du gras abdominal-poids vif varient de 1,9 à 3,5 et les 4 droites de régression, quand on utilise les valeurs brutes, sont différentes. Toutefois, une seule droite de régression peut être utilisée pour les 2 lignées X-33 et X-44.

Nos résultats confirment l'hypothèse selon laquelle les relations entre caractéristiques de carcasse du poulet dépendent du génotype considéré.
\end{abstract}

Mots clés : Sélection croissance, sélection engraissement, angle de poitrine, carcasse effilée, gras abdominal, poulet.

\section{Summary}

Growth and carcass characteristics of chickens form lines divergently selected for growth or fatness

Chickens from 4 experimental lines were raised in the same environment to 9 weeks of age. These lines were selected either for high or low growth rate (X-33 and X-44) or for high or low abdominal fat content (fat and lean lines). All the birds were weighed at 3,6 and 
9 weeks, breast angle was measured on living chickens at 9 weeks. Then, 32 cockerels per line were slaughtered, carcass breast angle was obtained, french dressed (effilé) carcasses and abdominal fat deposits were weighed.

Because of the very different genotypes, differences between lines were observed for all the measured characteristics. Between 3 and 9 weeks of age, weight gain of the lean birds was slightly but significantly higher than the gain of the fat birds. Average phenotypic correlations with live body weight were $.994, .59, .40, .29$, and .26 for effile carcass weight, abdominal fat weight, abdominal fat percentage, effilé carcass weight/live weight ratio and carcass breast angle, respectively.

Changes in effile carcass weight variations strictly followed those of live body weight and the 4 regression lines (effile weight on live weight) may be combined. Fatness variations showed specific patterns between lines. Correlation coefficients with breast angle were practically zero for fat and lean lines, and 0.45 for $X-33$ and $X-44$ lines. Allometric coefficients of abdominal fat weight on live weight varied from 1.9 (fat line) to 3.5 (lean line) and the 4 regression lines obtained with raw values are statistically different. However, a common regression line could be drawn for the $X-33$ and $X-44$ lines.

Our results corroborate the hypothesis of an effect of the chicken genotype on relationships between carcass characteristics.

Key words : Selection for growth, selection for fatness, breast angle, dressed carcass, abdominal fat, chicken.

\section{Introduction}

Deux expériences de sélection indépendantes l'une de l'autre ont été entreprises chez le poulet, la première pour explorer la possibilité de modifier génétiquement la forme de la courbe de croissance (RICARD, 1975), la seconde pour essayer d'obtenir des animaux génétiquement gras ou maigres (LecLerce et al., 1980). Les lignées du programme courbe de croissance ont été sélectionnées à partir de la souche «Bressepile » qui existait au Domaine I.N.R.A. du Magneraud en 1960. Nous avons utilisé ici la plus lourde (appelée X-33) et la plus légère (appelée X-44) dont les premières générations ont été obtenues en 1964. Jusqu'en 1980, la sélection portait à la fois sur les poids juvénile ( 8 semaines) et adulte (36 semaines). Depuis 1980, les effectifs ont été réduits et la sélection ne porte que sur le poids vif à 9 semaines. Les lignées "grasse » et «maigre » ont été obtenues en 1978 par sélection divergente à partir d'une population synthétique créée à la Station (BOYER, communication personnelle). A la première génération, le critère de sélection était l'écart du poids du gras abdominal à sa régression sur le poids vif à l'âge de 9 semaines. Aux générations suivantes, c'était le pourcentage gras abdominal/poids vif, également mesuré sur collatéraux à l'âge de 9 semaines. Pour maintenir une vitesse de croissance comparable entre les 2 lignées, les reproducteurs étaient choisis de façon à avoir un poids vif moyen à 9 semaines aussi voisin que possible. Les particularités métaboliques des lignées grasse et maigre ont été passées en revue récemment (Simon \& Leclerce, 1983) et l'évolution du pourcentage du dépôt gras abdominal pour les générations F1 à F7 a été présentée par Leclerce (1984).

Un essai a été mis en place pour comparer la vitesse de croissance de ces 4 lignées, leur conformation, le rendement à l'abattage "effilé » ainsi que l'importance du dépôt gras abdominal. Le but recherché était d'une part d'obtenir des valeurs comparatives pour des poulets de types très différents mais élevés dans les mêmes conditions de milieu, et d'autre part d'étudier les variations possibles, liées au génotype, des relations entre caractéristiques de carcasse. 


\section{Matériel et méthodes}

\section{A. Elevage des animaux}

Un échantillon représentatif de poulets des 4 lignées (grasse, maigre, lourde, légère), incubés dans le même incubateur et éclos le même jour, a été mis en élevage dans la même case de poussinière. Les animaux des lignées grasse et maigre correspondaient à la génération F7 et ceux des lignées lourde et légère à la génération F20. Tous les poulets ont donc subi les mêmes conditions de milieu. Ils ont été vaccinés contre la maladie de Marek à l'éclosion. De la tylosine a été ajoutée à l'eau de boisson durant les 3 premiers jours ainsi que pendant 24 heures au cours de la quatrième semaine. Ils ont reçu 24 heures de lumière durant les 3 premiers jours, puis 16 heures jusqu'au dixième jour, puis 12 heures jusqu'à l'abattage. Les principales caractéristiques de l'aliment utilisé, supplémenté en anti-coccidien, figurent dans le tableau 1.

\section{TABleaU 1}

Caractéristiques principales des aliments utilisés.

Main characteristics of the diets.

\begin{tabular}{|c|c|c|}
\hline & $\begin{array}{c}\text { Démarrage } \\
(0-3 \text { semaines })\end{array}$ & $\begin{array}{c}\text { Croissance } \\
\text { (3-9 semaines) }\end{array}$ \\
\hline Energie métabolisable $(\mathrm{MJ} / \mathrm{kg})$ & 13,0 & 12,8 \\
\hline Matières azotées totales $(\mathrm{g} / \mathrm{kg})$. & 239 & 191 \\
\hline Lysine $(\mathrm{g} / \mathrm{kg}) \ldots \ldots \ldots \ldots \ldots$ & 13,7 & 9,9 \\
\hline Méthionine + cystine $(\mathrm{g} / \mathrm{kg})$ & 10,1 & 7,9 \\
\hline Présentation $\ldots \ldots \ldots \ldots$ & Farine & Granulé $2,5 \mathrm{~mm}$ \\
\hline
\end{tabular}

\section{B. Caractéristiques mesurées}

Tous les animaux ont été pesés aux âges de 3,6 et 9 semaines, après un jeûne complet d'environ 16 heures. L'angle de poitrine a été mesuré à 9 semaines sur les poulets vivants à l'aide d'un anglemètre (RICARD, 1961). Dans la souche Bresse-pile, l'angle de poitrine est fortement corrélée à la compacité de la carcasse (RouviER \& RICARD, 1965 ; RICARD \& RouvieR, 1966). On peut considérer que c'est le meilleur critère de conformation de la carcasse (RICARD, 1980).

Immédiatement après la pesée 9 semaines, 32 coquelets par lignée ont été abattus, plumés après trempage dans un bac d'eau à $52^{\circ} \mathrm{C}$, et effilés (intestins et pancréas enlevés). Les carcasses effilées ont été pesées et une nouvelle mesure de l'angle de poitrine a été faite sur la carcasse. Le dépôt gras abdominal a été prélevé selon la technique habituelle de notre laboratoire : tissu gras qui tapisse la paroi 
abdominale et qui entoure l'intestin, le gésier et le ventricule succenturié. La carcasse effilée et le dépôt gras abdominal ont été exprimés en pourcentage du poids vif mesuré avant l'abattage.

\section{Calculs réalisés}

Les paramètres statistiques (moyenne et écart-type) ont été calculés pour tous les caractères mesurés. Une analyse de variance à un facteur a été faite pour chacun d'eux et les moyennes ont été comparées à l'aide du test de Newmann et Keuls (DAGNELIE, 1975) quand les variances étaient homogènes.

Pour les caractéristiques mesurées sur la carcasse, les corrélations phénotypiques ont été calculées séparément pour chaque lignée. Quand les 4 valeurs pouvaient être considérées comme homogènes, une valeur combinée pour l'ensemble des 4 lignées a été obtenue, par l'intermédiaire de la variable $\mathrm{z}$ de Fisher (DaGnelie, 1975).

Pour le poids de la carcasse effilée et du dépôt gras abdominal, les équations de régression sur le poids vif ont été calculées, d'abord sur les valeurs brutes puis sur les données transformées en logarithmes népériens. Les lignées ont ensuite été comparées à poids vif fixé, à l'aide d'une analyse de covariance (DAGNELIE, 1975). Par ailleurs, les calculs sur logarithmes permettent d'obtenir les coefficients d'allométrie.

\section{Résultats et discussion}

Les paramètres statistiques du poids vif et de l'angle de poitrine de tous les poulets présents à l'âge de 9 semaines figurent au tableau 2. Les paramètres des caractéristiques mesurées sur la carcasse des coquelets abattus (32 par lignée), ainsi que leur poids vif avant abattage, figurent au tableau 3. Les différences observées pour le poids vif et l'état d'egraissement s'expliquent aisément par le choix des lignées. La faible croissance des poulets X-44 entraîne une hétérogénéité des variances pour tous les caractères mesurés, à l'exception du rendement effilé/vif. De même, le fort état d'engraissement de la lignée grasse perturbe les comparaisons pour le poids brut ou le pourcentage du dépôt gras abdominal. Les comparaisons de moyennes figurant aux tableaux 2 et 3 tiennent compte de ces considérations.

Les corrélations phénotypiques entre les caractéristiques de carcasse sont indiquées dans le tableau 4. Les paramètres des équations de régression du poids de la carcasse effilée et du dépôt gras abdominal sur le poids vif figurent dans le tableau 5 et les résultats des analyses de covariance dans le tableau 6 .

Les lignées grasse et maigre ont des poids très voisins à 3 et 6 semaines. A 9 semaines, les poulets maigres de notre échantillon ont tendance à être plus lourds que les gras, la différence étant significative $(P<0,05)$ pour les poulettes (tabl. 2). Le gain de poids entre 3 et 9 semaines est donc plus important chez les poulets maigres, la différence étant significative pour les femelles. Ce résultat confirme qu'il existe une meilleure efficacité protéique chez le génotype «maigre » lié à une plus forte aptitude pour la croissance musculaire (LECLERCQ, 1983). Mais cette observation n'est pas corroborée par les mesures d'angle de poitrine, plus élevées pour 


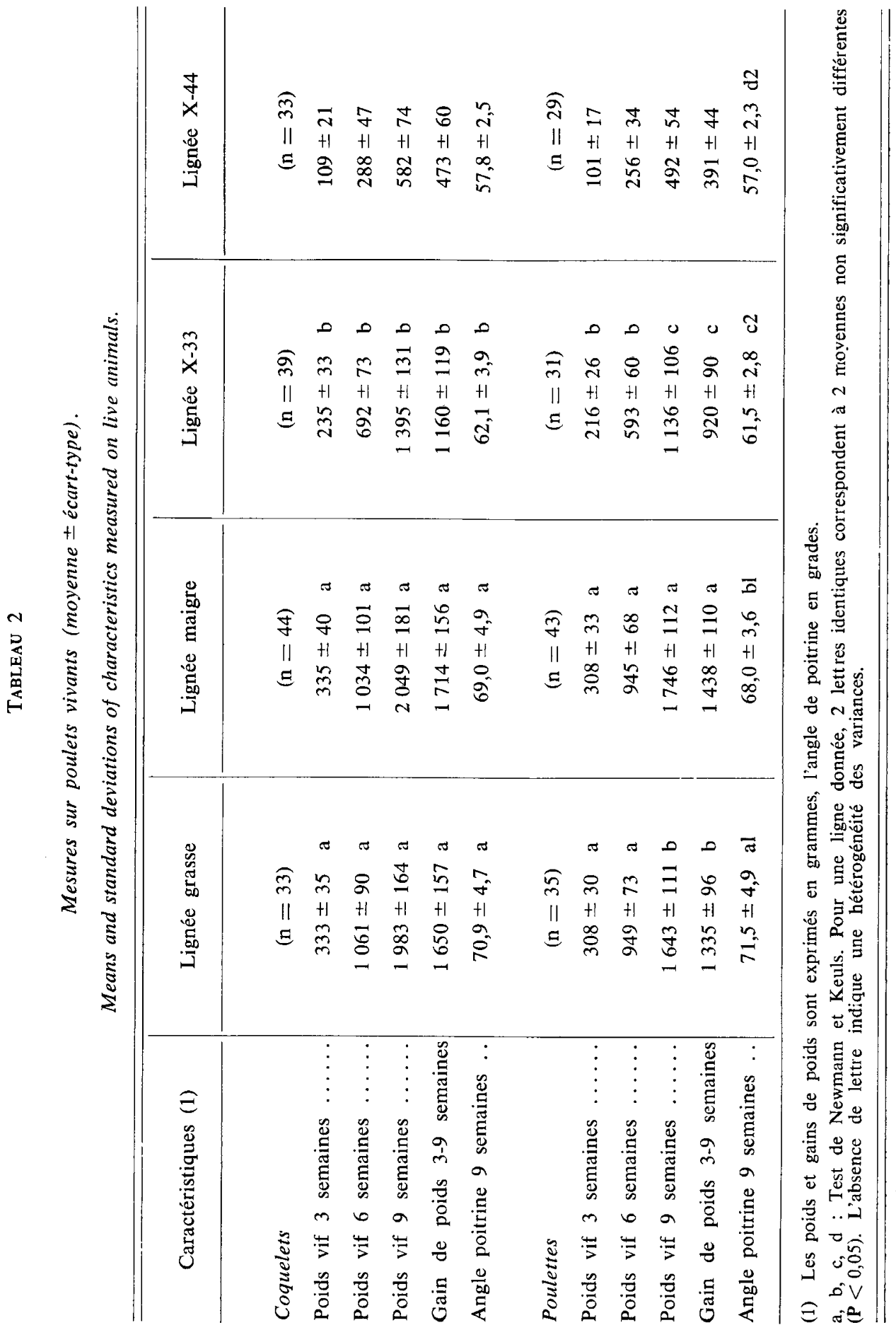




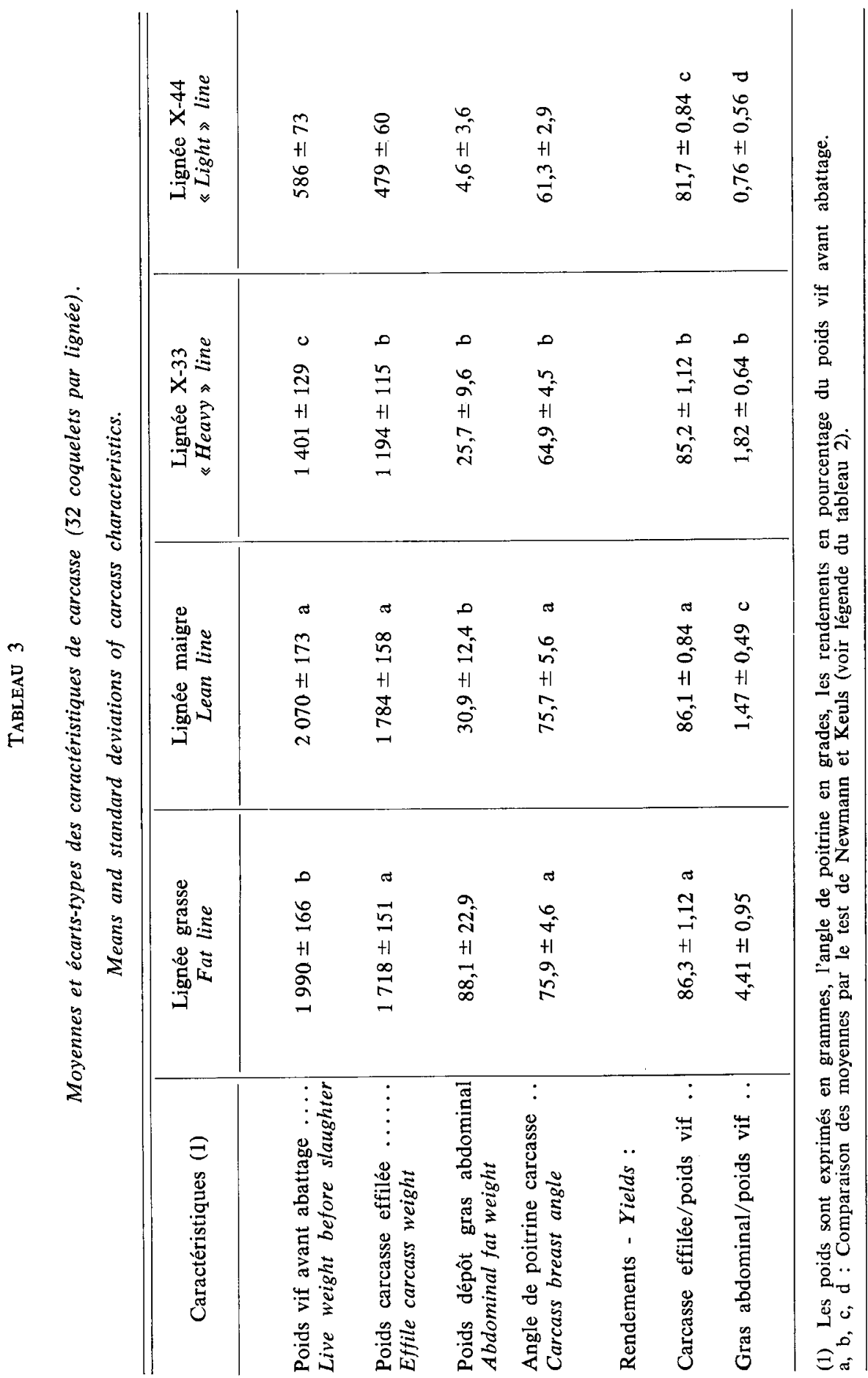


TABleaU 4

Corrélations phénotypiques entre les caractéristiques de carcasse (32 coquelets par lignée). Phenotypic correlations between carcass characteristics.

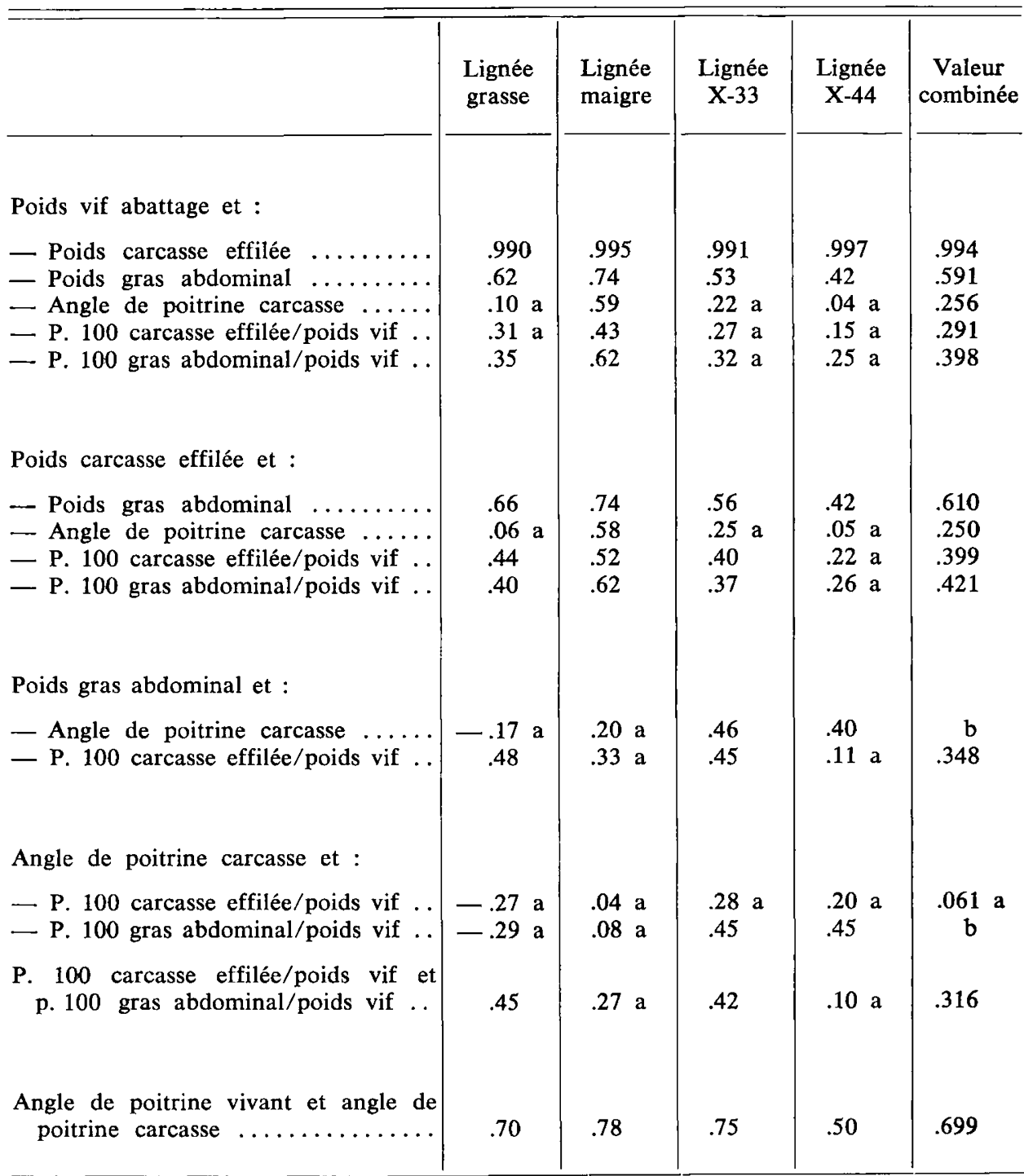

a : Valeur de la corrélation non significativement différente de zéro $(P<0,05)$.

b : Valeur combinée non calculée à cause de l'hétérogénéité des 4 corrélations intralignées. 


\section{TABLEAU 5}

Equations de régression du poids de la carcasse effilée et du dépôt gras abdominal sur le poids vif ( $y=a+b x$ où $x=$ poids vif avant abattage exprimé en grammes).

Regression equations of effile and abdominal fat weights on live weight.

\begin{tabular}{|c|c|c|c|c|c|c|}
\hline & \multicolumn{3}{|c|}{ Calculs sur valeurs brutes } & \multicolumn{3}{|c|}{ Calculs sur Log } \\
\hline & a & b & $s_{\mathrm{h}}$ & a & b (1) & $s_{b}$ \\
\hline 1. Poids carcasse effilée $(\mathrm{g})$ & & & & & & \\
\hline Lignée grasse $\ldots \ldots \ldots \ldots$ & $-81,6$ & 0,904 & 0,024 & $-0,511$ & 1,048 & 0,027 \\
\hline Lignée maigre $\ldots \ldots \ldots \ldots$ & $-93,0$ & 0,907 & 0,016 & $-0,531$ & 1,050 & 0,019 \\
\hline Lignée $X-33$ & $-44,5$ & 0,884 & 0,022 & $-0,454$ & 1,040 & 0,025 \\
\hline Lignée X-44 & $-5,7$ & 0,827 & 0,012 & $-0,272$ & 1,011 & 0,014 \\
\hline 2. Poids gras abdominal (g) & & & & & & \\
\hline Lignée grasse & $-83,4$ & 0,086 & 0,020 & $-10,13$ & 1,920 & 0,475 \\
\hline Lignée maigre . . & $-79,2$ & 0,053 & 0,009 & $-23,06$ & 3,461 & 0,642 \\
\hline Lignée $\mathrm{X}-33$. & $-29,2$ & 0,039 & 0,011 & $-18,22$ & 2,954 & 0,699 \\
\hline Lignée $X-44 \quad \ldots \ldots \ldots \ldots$ & $-7,6$ & 0,021 & 0,008 & $-13,32$ & 2,292 & 0,906 \\
\hline
\end{tabular}

(1) Représente également le coefficient d'allométrie.

le génotype «gras» (différence significative sculement chez les femelles). Ce dernier résultat pourrait être la conséquence d'une plus forte couche de gras sous-cutané (RICARD et al., 1983).

La différence entre la vitesse de croissance des lignées X-33 et X-44 est considérable puisqu'à 9 semaines le rapport des poids vifs est de 2,4. Dix générations plus tôt, ce même rapport était de l'ordre de 1,9 (RICARD, 1975). Mais on ne peut pas dire que la lignée X-33 soit «lourde» si on la compare aux souches modernes de type Cornish. Les résultats que nous observons montrent que la croissance des lignées grasse et maigre correspond à celle des souches de type femelle-chair (White-Rock par exemple); la croissance de la lignée X-33 se rapproche de celle des souches "label »; la croissance de la lignée X-44 peut se comparer à celle des souches de type Leghorn. 


\section{TABLEAU 6}

Comparaison des lignées par analyse de covariance, à poids vif fixé.

Line comparisons by covariance analyses, with live body weight being held constant.

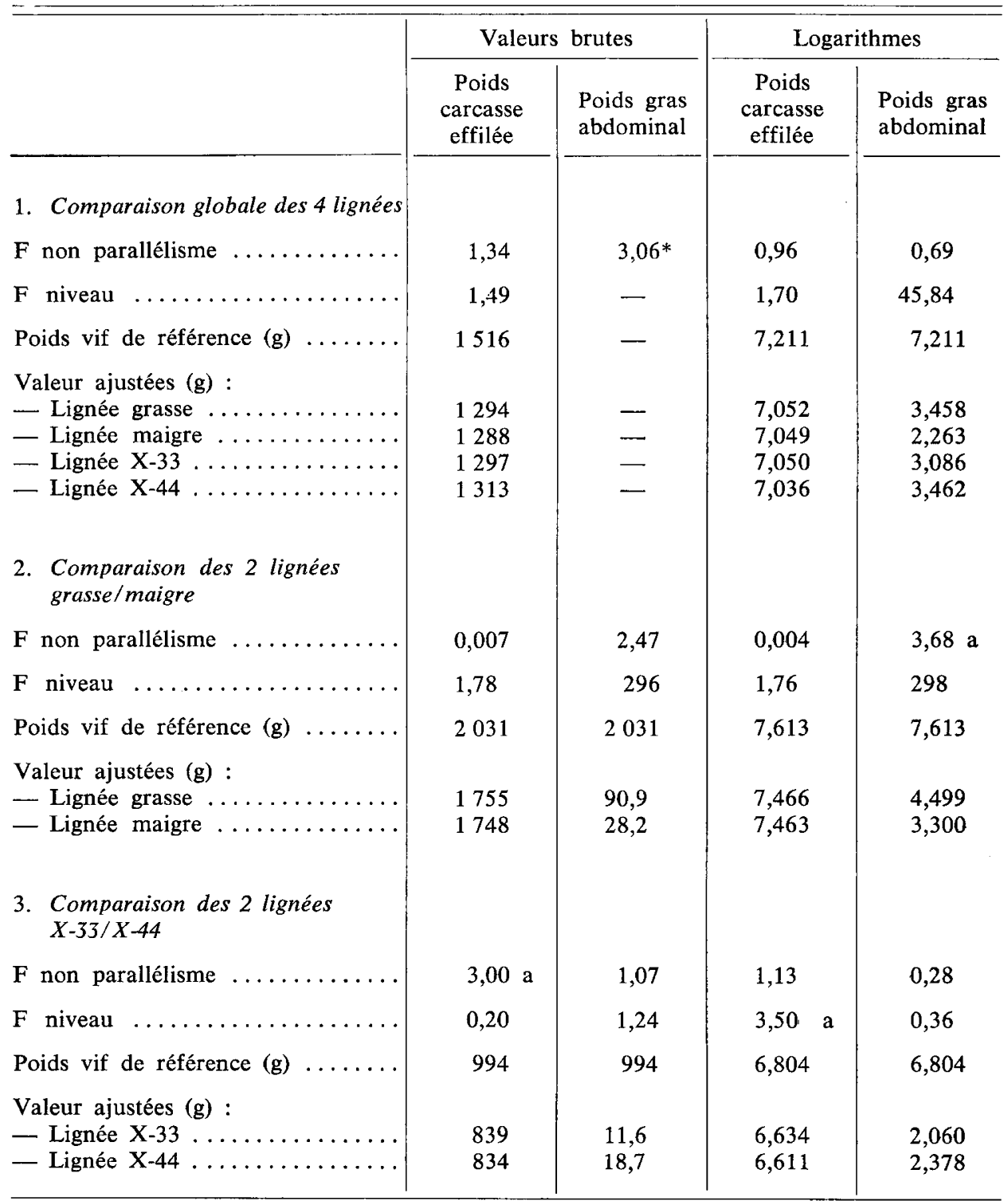

* : Valeur significative au seuil 5 p. 100, la suite des calculs n'a donc pas été faite.

a : La valeur de F significative au seuil 5 p. 100 serait de 4,0. 
L'angle de poitrine des poulets X-33 et X-44 est faible quand on les compare avec les souches modernes. Cela s'explique par l'origine génétique des animaux (la souche Bresse-pile est de type longiligne) et le fait qu'aucune sélection n'a été faite sur ce caractère. Hormis le cas de la souche maigre, l'angle de poitrine apparaît faiblement corrélé au poids vif (et au poids de carcasse). La corrélation entre les 2 mesures prises sur le vivant et sur carcasse est de l'ordre de 0,7 (dernière ligne du tabl. 4), ce qui est significativement supérieur aux valeurs trouvées dans le travail de LIEN (1973). La précision de la mesure est meilleure sur la carcasse que sur le poulet vivant (Rouvier \& Ricard, 1966). La présence des plumes et les mouvements du poulet vivant, même maintenu sur une table, peuvent expliquer cette moindre précision. Ils expliquent aussi les différences observées entre les 2 mesures : comme dans plusieurs expériences précédentes, nous trouvons généralement la mesure de carcasse plus élevée (RICARD, 1970 et données non publiées) ; LIEN (1973) trouve au contraire une valeur plus faible sur carcasse que sur le poulet vivant. Les corrélations entre l'angle de poitrine et le rendement poids effilé/poids vif ne sont pas significativement différentes de zéro (tabl. 4). Au contraire, le rendement poids éviscéré/poids vif est généralement corrélé de façon positive et significative avec l'angle de poitrine (RICARD, 1980).

Les variations de poids de la carcasse effilée suivent celles du poids vif, puisqu'on observe une très forte corrélation (supérieure à 0,99 ) entre ces 2 caractères (tabl. 4). Les différences entre lignées pour le rendement poids effilé/poids vif s'expliquent entièrement par les variations de poids vif. Le coefficient d'allométrie (tabl. 5) est légèrement supérieur à 1 et très voisin de ce qu'ont observé GREY et al. (1982) pour l'allométrie poids éviscéré/poids vif. La comparaison de nos 4 lignées par analyse de covariance montre que les 4 droites de régression peuvent être confondues en une seule qui s'écrit :

avec $\mathrm{r}=0,992$.

$$
\text { Poids effilé (en } \mathrm{g})=-60,9+0,904 \text { poids vif (en g) }
$$

Les variations de poids, et du pourcentage, du dépôt gras abdominal sont particulièrement importantes (tabl. 3), qu'il s'agisse du coefficient de variation intra-lignée compris entre 0,2 et 0,8 ), ce qui marque la forte variabilité biologique de ces caractères, ou des différences entre moyennes de lignées, différences liées au choix des génotypes étudiés. Elles permettent de comprendre que de nombreux auteurs aient mis en évidence des différences significatives entre souches commerciales (voir par exemple : VAN Middelkoop et al., 1977 ; Griffiths et al., 1978 ; Scheele et al., 1981 ; Ehinger \& Seeman, 1982).

Dans la présente étude, nous retrouvons l'écart important, obtenu grâce à la sélection directe, entre les lignées grasse et maigre : les résultats présentés par LECLERCQ (1984) montrent en effet que les pourcentages du dépôt gras abdominal étaient respectivement de 3,7 et 1,3 à la génération $F 6$, de 3,6 et 0,9 à la génération F7, alors que nous observons ici 4,4 et 1,5 . Malgré un poids vif plus faible, les coquelets de la lignée $\mathrm{X}-33$ ont un poids de gras abdominal significativement non différent de ceux de la lignée "maigre », ce qui entraîne un pourcentage par rapport au poids vif plus élevé (tabl. 3). Le dépôt gras abdominal des coquelets X-44 est voisin de ce qu'on trouve chez des poulets de type Leghorn (Allain \& Simon, 1982 ; MARCH, 1984).

L'analyse plus détaillée de l'état d'engraissement montre un comportement variable selon les lignées. Bien que les 4 valeurs soient homogènes (tabl. 4), les corrélations entre poids vif (ou poids effilé) et poids du gras abdominal (ou pourcen- 
tage par rapport au poids vif) sont plus élevées dans le cas des lignées grasse et maigre que dans le cas des lignées $\mathrm{X}-33$ et X-44. La valeur plus faible obtenue pour la lignée X-44 peut s'expliquer par la faible quantité du tissu adipeux disséqué d'où une imprécision plus grande en valeur relative. Mais le type génétique peut également jouer un rôle. Ainsi, Griffiths et al. (1978) citent des corrélations allant de 0 à 0,56 , selon la souche étudiée, entre le poids vif et le pourcentage de gras abdominal. De même, van MidDELKoop et al. (1977) obtiennent entre les mêmes caractères des valeurs allant de 0,09 à $0,33 \mathrm{chez}$ les coquelets et de $-0,16$ à 0,58 chez les poulettes.

Les corrélations entre l'angle de poitrine et le poids, ou le pourcentage, du dépôt gras abdominal sont hétérogènes : non significativement différentes de zéro pour les 2 lignées grasse et maigre, elles sont de l'ordre de 0,45 pour les lignées X-33 et X-44. Sur des poulets de type Rhode-Island âgés de 9 semaines, JENKINS et al. (1982) ont trouvé une corrélation pratiquement nulle entre angle de poitrine et engraissement, ce qui confirme la variabilité de cette relation selon le type d'animaux étudié. Les corrélations entre les pourcentages effilé/vif et gras abdominal/vif sont homogènes mais plus élevées pour les lignées grasse et $\mathrm{X}-33$ que pour les lignées maigre et X-44. Une autre preuve de l'influence du génotype sur les relations entre caractéristiques de carcasse est fournie par la comparaison des coefficients d'allométrie gras abdominal-poids vif, plus élevés pour les lignées maigre et X-33 que pour les lignées grasse et X-44 (tabl. 5).

Quand on exprime les données (poids vif et poids du gras abdominal) en valeurs brutes, les 4 droites de régression ne peuvent pas être considérés comme parallèles (tabl. 6). Mais elles peuvent l'être quand on compare uniquement les 2 lignées grasse et maigre, ou uniquement les 2 lignées $\mathrm{X}-33$ et X-44. Les équations de régression obtenues pour les lignées grasse et maigre sont distinctes et ressemblent à celles obtenues par BECKER et al. (1981) pour des coquelets abattus à l'âge de 8 semaines au poids vif moyen de $2112 \mathrm{~g}$. Dans la présente expérience, pour un même poids vif théorique de $2031 \mathrm{~g}$, le dépôt gras abdominal serait de $9.1 \mathrm{~g}$ pour la lignée grasse et de $28 \mathrm{~g}$ pour la lignée maigre. Pour les 2 lignées X-33 et X-44, les 2 droites de régression peuvent être confondues. Les ordonnées à l'origine et les coefficients de régression se rapprochent des valeurs obtenues par VAN MIDDELKooP et al. (1977) sur des coquelets plus jeunes (6 $1 / 2$ semaines) à un poids vif moyen de $1746 \mathrm{~g}$. Dans notre cas, l'équation de régression commune aux 2 lignées X-33 et X-44 s'écrit :

avec $\mathrm{r}=0,50$.

$$
\text { poids gras abdominal }(\mathrm{g})=-19,4+0,035 \text { poids vif }(\mathrm{g})
$$

Quand on exprime les données en logarithmes (dernière colonne du tableau 6), les variations de poids s'estompent et les 4 droites de régression peuvent être considérées comme parallèles, mais les niveaux restent significativement différents. Nous observons que pour un même poids vif fixé les poulets génétiquement petits (X-44) auraient des dépôts adipeux aussi développés que des poulets génétiquement gras et un peu plus développés que les poulets de la lignée X-33. Il serait intéressant d'approfondir ce résultat en étudiant, sur des effectifs plus nombreux et sur d'autres échantillons, les variations de l'état d'engraissement pour des lignées qui présentent des vitesses de croissance différentes. 


\section{Références bibliographiques}

Allain X., Simon J., 1982. Inefficacité d'une suralimentation imposée dès la naissance pour modifier le comportement alimentaire, la croissance et la composition corporelle ultérieure du poulet. Reprod. Nutr. Dévelop., 22, 27-40.

Becker W.A., Spencer J.V., Mirosh L.W., Verstrate J.A., 1981. Abdominal and carcass fat in five broiler strains. Poult. Sci., 60, 693-697.

Dagnelie P., 1975. Théorie et méthodes statistiques. Vol. 2 : Les méthodes de l'inférence statistique. $2^{\mathrm{e}}$ éd., 463 pp., Les Presses Agronomiques, Gembloux.

Ehinger F., Seeman G., 1982. Einfluss von Futter, Alter, und Geschlecht auf die Mastleistung un die Schlachtkörperqualität von Broilern verschiedener Herkunft. 2-Verfettungsgrad. Arch. Geflügelk., 46, 177-188.

Grey T.C., Robinson D., Jones J.M., 1982. Effect of age and sex on the eviscerated yield, muscle and edible offal of a commercial broiler strain. Br. Poult. Sci., 23, 289-298.

Griffiths L., LeESon S., Summers J.D., 1978. Studies on abdominal fat with four commercial strains of male broiler chicken. Poult. Sci., 57, 1198-1203.

JenKinS J.L., Lewin T.L., BriLes C.O., 1982. Effects of two dietary protein levels on quantitative traits in growing dual purpose chickens. Poult. Sci., 61, 1382 (abstract).

LECLERCQ B., 1983. The influence of dietary protein content on the performance of genetically lean and fat growing chickens. Br. Poult. Sci., 24, 581-587.

Leclerce B., 1984. Adipose tissue metabolism and its control in birds. Poult. Sci., 63, 2044-2054.

LeClerCQ B., Blum J.C., Boyer J.P., 1980. Selecting broilers for low or high abdominal fat : initial observations. Br. Poult. Sci., 21, 107-113.

LIEN S., 1973. Genetic and phenotypic parameters for live weight and some quality characters in broiler (en Norvégien). Meld. Norg. LandbrHogsk., 52 (24), 1-18.

MARCH B.E., 1984. Plasma triglyceride and glucose clearance in broiler-type and white Leghorn chickens with different degrees of adiposity. Poult. Sci., 63, 1586-1593.

RiCARD F.H., 1961. Note sur les méthodes d'estimation de l'angle de poitrine des poulets de chair. Ann. Zootech., '10, 69-72.

RICARD F.H., 1970. Etude d'un gène de nanisme lié au sexe chez la poule. IV. Observations sur la croissance et les caractéristiques de carcasse du jeune poulet. Ann. Génét. Sél. Anim., 2, 19-31.

Ricard F.H., 1975. Essai de sélection sur la forme de la courbe de croissance chez le poulet. Ann. Génét. Sél. Anim., 7, 427-443.

RiCaRD F.H., 1980. Carcass conformation of poultry and game birds. In MEAD G.C., Freeman B.M. (ed.), Meat quality in poultry and game birds, 31-50, British Poultry Science Ltd, Edinburgh.

Ricard F.H., LECLERCQ B., Touraille C., 1983. Selecting broilers for low or high abdominal fat : distribution of carcass fat and quality of meat. Br. Poult. Sci., 24, 511-516.

RICARD F.H., RouviER R., 1966. Etude des mesures de conformation du poulet. 4-Variabilité génétique des mensurations de carcasse et d'un écart viande/os chez des coquelets Bress-pile. Ann. Zootech., 15, 197-209.

Rouvier R., Ricard F.H., 1965. Etude des mesures de conformation du poulet. 2-Recherche des composantes de la variabilité morphologique du poulet vivant. Ann. Zootech., 14, 213-227.

Rouvier R., Ricard F.H., 1966. Etude des mesures de conformation du poulet. 3-Analyse de la variabilité phénotypique des mensurations de carcasse de coquelets Bresse-pile. Ann. Zootech., 15, 67-84.

Scheele C.W., Van Schagen P.J.W., Ten Have H.G.M., 1981. Abdominal and total fat content of three broiler strains at two ages affected by nutritional factors. In Mulder R.W., Scheele C.W., WeErKamP C.H. (ed.), Quality of poultry meat, 397-407, Spelderholt Institute, Beekbergen.

Simon J., Leclerce B., 1983. Relation entre insulinémie et adiposité dans deux lignées de poulets rendus maigres ou gras par sélection. Reprod. Nutr. Dévelop., 23, 443-451.

VAN Middelkoop J.H., KuIT A.R., Zegward A., 1977. Genetic factors in broiler fat deposition. In BooRMAN K.N., WILSON B.J. (ed.), Growth and poultry meat production, 131-143, British Poultry Science Ltd, Edinburgh. 